\title{
GAIA Level 1 Dysfunctional Second Stage of Labor
}

National Cancer Institute

\section{Source}

National Cancer Institute. GAIA Level 1 Dysfunctional Second Stage of Labor. NCI

Thesaurus. Code C128719.

GAIA Level 1 Dysfunctional Second Stage of Labor is defined by three criteria. For nulliparous women: first, full dilation of the cervix $(10 \mathrm{~cm}$ dilated, or no palpable cervix around the presenting part of the fetus); second, the onset of the active stage of labor (active maternal effort (i.e. pushing) or visible baby); third, at least one of the following requirements must be met: a) Greater than 2 hours of pushing; OR b) The use of an instrument (forceps or vacuum/ventouse) for the indication of dystocia; OR c) Cesarean delivery for the indication of dystocia. For multiparous women: first, full dilation of the cervix (10 cm dilated, or no palpable cervix around the presenting part of the fetus); second, the onset of the active stage of labor (active maternal effort (i.e. pushing) or visible baby); third, at least one of the following requirements must be met: a) Greater than 1 hour of pushing; OR b) The use of an instrument (forceps or vacuum/ventouse) for the indication of dystocia; OR c) Cesarean delivery for the indication of dystocia. 